\title{
Angiotensin Converting Enzyme Inhibitors: Should They be Used Early Post Myocardial Infarction?
}

\author{
Bertram Pitt, MD \\ University of Michigan School of Medicine, Ann Arbor, \\ Michigan, USA
}

Angiotensin II (ATII) levels are elevated in the myocardium after myocardial damage independent of serum levels [1]. ATII results in an increase $\mathrm{NAD}(\mathrm{P}) \mathrm{H}$ vascular oxidase, the most important vascular source of reactive oxygen species (ROS); destruction of nitric oxide (NO); endothelial dysfunction; vascular inflammation; vascular remodeling; myocardial hypertrophy and collagen formation; the progression of atherosclerosis; atherosclerotic plaque instability; thrombosis and impaired fibrinolysis. All of the adverse effects of ATII appear to be mediated through the AT1 receptor and can be prevented by angiotensin converting enzyme inhibitors (ACE-I) or angiotensin receptor antagonists (ARB's) providing a strong scientific basis for the clinical use of ACE-I or ARB's in patients with acute myocardial infarction (MI).

A meta analysis of all randomized trials of ACE-Is post MI [2] suggests that a relatively uniform beneficial effect on mortality/morbidity occurs in all subgroups including age, gender, baseline use of aspirin, beta blockers, and reperfusion status. The valiant trial [3] comparing the ACE-I captopril to the ARB valsartan showed that blocking the AT1 receptor by valsartan was as effective as inhibiting the conversion of Angiotensin I to ATII by captopril. Inhibition or blockade of ATII post MI has been suggested to be beneficial regardless of the extent of myocardial damage and the time from onset of MI, although the benefits appear greater in those early post MI with signs of heart failure and or a reduced left ventricular ejection fraction (LVEF) [2].

It is therefore surprising, that Voors et al. [4] in a pooled analysis of three randomized placebo controlled trials of ACE-I use within 9 hours of onset of mainly a first anterior MI find that although ACE-I reduce the 3 month incidence of hospitalization for HF they double the 3 month re-infarction rate from 3.6 to $7.0 \%$, $p=0.032$. In those with a relatively large MI and a reduced LVEF the favorable effects of ACE-Is on ventricular remodeling appear to outweigh the risks of reinfarction. However, in those with a relatively small MI the risk or re-infarction appears to outweigh the benefits such that the net effect is unfavorable. Of interest was their observation that the incidence of reinfarction post MI was related to ACE-I induced hypotension. Hypotension post MI could trigger ischemia, platelet activation, and thrombosis. Patients with relatively small MIs often have multiple areas of jeop- ardized myocardium aside from their infarct related artery. These areas of jeopardized myocardium are served by narrowed arteries or collateral vessels. Perfusion pressure beyond the narrowed artery or collateral vessel is lower than systemic blood pressure. A drop in systemic blood pressure of only a few $\mathrm{mmHg}$ could therefore trigger myocardial ischemia with all of its adverse consequences including re-infarction and possibly sudden cardiac death. Hypotension might also explain the failure of valsartan to add to the benefical effects of captopril post MI in the valiant trial [3].

Thus, the observation that ACE-Is increase the incidence of re-infarction when given early post MI is scientifically plausible and could have an important impact upon our current approach to treating patients early post MI. However, before altering our current approach it might be useful to carefully review the basis for the findings of Voors et al. [4]. The definition of re-infarction in the studies they reviewed was not prospectively determined but left to individual investigators. One would have hoped for a prospectively defined definition based upon a re-elevation of troponin. Thus, while scientifically plausible the authors observations will require further confirmation by a prospective randomized study before their hypothesis is proven. However, while I will continue to use an ACE-I or an ARB in the early hours post MI, especially in patients with evidence of $\mathrm{HF}$ and or a low LVEF, I will be more cautious and would start with a low dose of captopril, especially in patients with a borderline systemic blood pressure and in those with a relatively small MI, and only up-titrate to target doses of captopril or switch to a longer acting ACE-I or ARB if blood pressure is maintained over the first 24-48 hours. The observations of Voors et al. [4] also suggest careful attention to the effect of any therapeutic agent on blood pressure considered for use during the early hours post MI.

\section{References}

1. Dzau VJ. Tissue rennin-angiotensin system in myocardial hypertrophy and failure. Arch Intern Med 1993;153: 937.

2. ACE Inhibitor Myocardial Infarction Collaborative Group: Indications for ACE Inhibitors in the early treatment of acute myocardial infarction: Systematic overview of 
individual data from 100,000 patients in randomized trials. Circulation 1998;97:2202.

3. Pfeffer MA. Effects of valsartan relative to captopril in patients with myocardial infarction complicated by heart failure and/or left ventricular dysfunction. Eng $\mathrm{J} \mathrm{Med}$ 2003;349:1843.
4. Voors AA, de Kam PJ, van den Berg MP, et al. Acute administration of angiotenin converting enzyme inhibitors in thrombolysed myocardial infarction patients is associated with a decreased incidence of heart failure, but an increased re-infarction risk. Cardiovasc Drugs and Ther 2005;19:119124. 